\title{
Speed Sensorless Control of a Doubly Fed Induction Motor Drives using MRAS Estimator
}

\author{
Djamila Cherifi", Yahia Miloud
}

Electrical Engineering Department, Moulay Tahar University, Saida, Algeria

Email address:

d_cherifi@yahoo.fr (D. Cherifi)

${ }^{*}$ Corresponding author

To cite this article:

Djamila Cherifi, Yahia Miloud. Speed Sensorless Control of a Doubly Fed Induction Motor Drives using MRAS Estimator. International Journal of Electrical Components and Energy Conversion. Vol. 4, No. 1, 2018, pp. 1-12. doi: 10.11648/j.ijecec.20180401.11

Received: December 25, 2017; Accepted: January 10, 2018; Published: February 23, 2018

\begin{abstract}
In this paper, direct vector control by rotor flux orientation for doubly fed induction motor without mechanical sensor based on the MRAS estimator is discussed, this method consists in developing two models one of reference and the other adjustable for the estimation of the two components of the rotor flux from the measurement of currents, statoric and rotor voltages respectively, the speed estimated is obtained by canceling the difference between the rotor flux of the reference model and the adjustable one, while using the theory of hyperstability to obtain the adaptive mechanism the simulation results are presented to validate the proposed method.
\end{abstract}

Keywords: Doubly Fed Induction Motor (DFIM), Field Oriented Control (FOC), Sensorless Speed Control, Estimator, MRAS

\section{Introduction}

The doubly fed induction machine (DFIM) is a very attractive solution for variable-speed applications such as electric vehicles and electrical energy production. Obviously, the required variable-speed domain and the desired performance depend on the application, [1], [2].

The DFIM has some distinct advantages compared to the conventional squirrel-cage machine. The DFIM can be fed and controlled stator or rotor by various possible combinations. Indeed, the input-commands are done by means of four precise degrees of control freedom relatively to the squirrel cage induction machine where its control appears quite simpler, [3], [4].

However, these advantages have long been inhibited by the complexity of the control, [5]. In order to obtain an DFIM having similar performance to a DC machine where there is a natural decoupling between the magnitude controlling the flux (the excitation current) and the magnitude related to the torque (the Armature current) [6], [7]. Several methods are used to control the induction motor among which the vector control (field oriented control) that also the decoupling between the torque and the flux, in order to obtain an independent control of torque and flux like DC motor, [8].
But, the knowledge of the rotor speed is necessary, this necessity requires additional speed sensor which adds to the cost and the complexity of the drive system. Over the past few years, ongoing research has concentrated on the elimination of the speed sensor at the machine shaft without deteriorating the dynamic performance of the drive control system. The advantages of speed sensorless induction motor drives are reduced hardware complexity and lower cost, reduces size of the drive machine, elimination of the sensor cable, better noise immunity, increased reliability and less maintenance requirements, [9].

In order to achieve good performance of sensorless vector control, different speed estimation schemes have been proposed, and a variety of speed estimators exist nowdays. Such as direct calculation method, model reference adaptive system (MRAS), Extended Kalman Filters (EKF), Extended Luenberger observer (ELO), sliding mode observer ect...

Among different rotor speed estimation techniques, model reference adaptive systems schemes are the most common strategies employed due to their relative simplicity and low computational effort,[5], [9].

This paper is organized as follows. Section 2 dynamic model of a DFIM is reported; principle of field-oriented controller is given in Section 3. The proposed solution is 
presented in Section 4. In Section 5, results of simulation tests are reported. Finally, Section 6 draws conclusions.

\section{Dynamic Model of Dfim}

By referring to a rotating reference frame, denoted by the superscript $(d, q)$, the dynamic model of a DFIM can be expressed by, [10]:

$$
\left\{\begin{array}{l}
\frac{d i_{s d}}{d t}=-a_{1} i_{s d}+\left(a \omega+\omega_{s}\right) i_{s q}+a_{3} i_{r d}+a_{5} \omega i_{r q}+b_{1} v_{s d}-b_{3} v_{r d} \\
\frac{d i_{s q}}{d t}=-\left(a \omega+\omega_{s}\right) i_{s d}-a_{1} i_{s q}-a_{5} \omega i_{r d}+a_{3} i_{r q}+b_{1} v_{s q}-b_{3} v_{r q} \\
\frac{d i_{r d}}{d t}=a_{4} i_{s d}-a_{6} \omega i_{s q}-a_{5} \omega i_{r d}+\left(\omega_{s}-\frac{\omega}{\sigma}\right) i_{r q}-b_{3} v_{s d}+b_{2} v_{r d} \\
\frac{d i_{r d}}{d t}=-a_{6} \omega i_{s d}-a_{4} i_{s q}-\left(\omega_{s}-\frac{\omega}{\sigma}\right) i_{r d}-a_{5} \omega i_{r q}-b_{3} v_{s q}+b_{2} v_{r q} \\
\frac{d \omega}{d t}=\frac{p^{2} L_{m}}{J}\left(i_{s q} i_{r d}-i_{s d} i_{r q}\right)-\frac{f}{J} \omega-\frac{p}{J} C_{r}
\end{array}\right.
$$

Where:

$$
\begin{gathered}
a=\frac{1-\sigma}{\sigma}, a_{1}=\frac{R_{s}}{\sigma L_{s}}, a_{2}=\frac{R_{r}}{\sigma L_{r}}, a_{3}=\frac{R_{r} \cdot M}{\sigma L_{r} L_{s}}, \\
a_{4}=\frac{R_{s} M}{\sigma L_{s} L_{r}}, \\
a_{5}=\frac{M}{\sigma L_{s}}, a_{6}=\frac{M}{\sigma L_{r}}, b_{1}=\frac{1}{\sigma L_{s}}, b_{2}=\frac{1}{\sigma L_{r}}, b_{3}=\frac{M}{\sigma L_{s} L_{r}}, \\
\sigma=1-\frac{M^{2}}{L_{s} L_{r}}
\end{gathered}
$$

The mathematical model for the mechanical parts is written as the following state equations:

$$
T_{e m}=J . \frac{d \Omega}{d t}+f \Omega+T_{r}
$$

Where

$$
\Omega=\frac{\omega}{p}
$$

$j$ is the moment of inertia of the revolving parts, $f$ is the coefficient of viscous friction, arising from the bearings and the air flowing over the motor, and $T_{r}$ is the load torque, $p$ is the number of pole pairs.

The electromagnetic torque is expressed by:

$$
T_{e m}=p M\left(i_{s q} i_{r d}-i_{s d} i_{r q}\right)
$$

\section{Principle of Field Oriented Controller}

The main objective of the vector control DFIM is, as in
DC machines, to independently control the torque and the flux; this is done by using a $d-q$ rotating reference frame synchronously with the rotor flux space vector. The d-axis is then aligned with the rotor flux space vector [5]. Under this condition we get:

$$
\phi_{\mathrm{rd}}=\phi_{\mathrm{r}} \text { and } \phi_{\mathrm{rq}}=0
$$

Figure 1 shows the structure for the rotor field orientation on the $d$-q axis

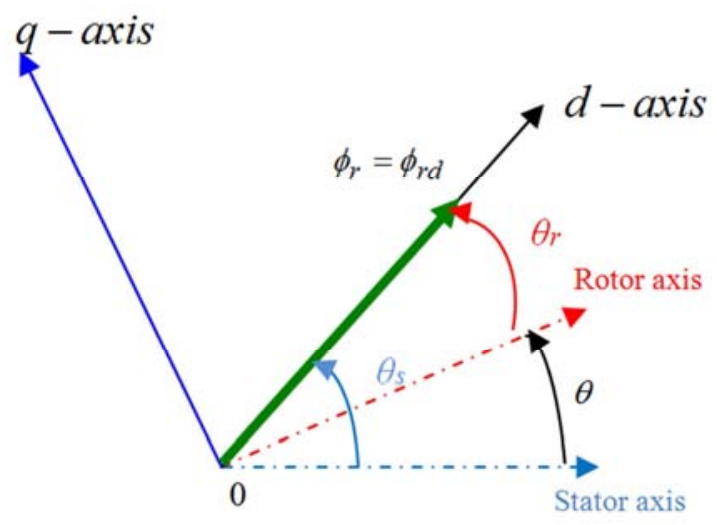

Figure 1. Rotor field orientation on the d-axis.

Park's relations linking the different main quantities of the DFIM are, [6].

$$
\left\{\begin{array}{l}
v_{s d}=R_{s} i_{s d}+\frac{d \phi_{s d}}{d t}-\omega_{s} \cdot \phi_{s q} \\
v_{s q}=R_{s} i_{s q}+\frac{d \phi_{s q}}{d t}+\omega_{s} \cdot \phi_{s d} \\
v_{r d}=R_{r} i_{r d}+\frac{d \phi_{r d}}{d t}-\omega_{r} \cdot \phi_{r q} \\
v_{r q}=R_{r} i_{r q}+\frac{d \phi_{r q}}{d t}+\omega_{r} \cdot \phi_{r d}
\end{array}\right.
$$

\subsection{The Equations linking the flux Are}

$$
\left\{\begin{array}{l}
\phi_{s d}=L_{s} i_{s d}+M i_{r d} \\
\phi_{s q}=L_{s} i_{s q}+M i_{r q} \\
\phi_{r d}=L_{r} i_{r d}+M i_{s d} \\
\phi_{r q}=L_{r} i_{r q}+M i_{s q}
\end{array}\right.
$$

He comes then:

$$
\phi_{r q}=0 \Leftrightarrow\left\{\begin{array}{l}
i_{r q}=-\frac{M}{L_{r}} i_{s q} \\
i_{s q}=-\frac{L_{r}}{M} i_{r q}
\end{array}\right.
$$

A relation between the quadrature component of the stator flux and the current $i_{r q}$ can be formulated, and a relation between the electromagnetic couple and the same current is written as below 


$$
\begin{aligned}
& \phi_{r d}=\phi_{r} \Leftrightarrow i_{r d}=\frac{1}{L_{r}}\left(\phi_{r}-M i_{s d}\right) \\
& \left\{\begin{array}{l}
\phi_{s q}=-\sigma \frac{L_{s} L_{r}}{M} i_{r q} \\
C_{e m}=p \cdot M\left(i_{s q} \cdot i_{r d}-i_{r q} \cdot i_{s d}\right)=-p \cdot \phi_{r d} \cdot i_{r q}
\end{array}\right.
\end{aligned}
$$

\subsection{Current Control and Compensation Term}

The following method was introduced by D. LECOQ. It requires the use of four current correctors, [6], [10].

In order to obtain a good decoupling between the magnitudes according to the axes $\mathrm{d}$ and $\mathrm{q}$, it defines new transformed voltages as follows

$$
\left\{\begin{array}{l}
v_{c, s d}=v_{s d}-\frac{M}{L_{r}} v_{r d} \\
v_{c, r d}=v_{r d}-\frac{M}{L_{s}} v_{s d}
\end{array}\right.
$$

Then, the model (1) becomes:

$$
\begin{aligned}
& \frac{d i_{s d}}{d t}=\frac{1}{\sigma L_{s}}\left(-R_{s} i_{s d}+\left(\omega_{s} \sigma+\omega(1-\sigma)\right) L_{s} i_{s q}+\frac{M R_{r}}{L_{r}} i_{r d}+\omega M \cdot i_{r q}\right)+\frac{1}{\sigma \cdot L_{s}} v_{c, s d} \\
& \frac{d i_{s q}}{d t}=\frac{1}{\sigma L_{s}}\left(-\left(\omega_{s} \sigma+\omega(1-\omega)\right) L_{s} i_{s d}-R_{s} i_{s q}-\omega M \cdot i_{r d}+\frac{M R_{r}}{L_{r}} i_{r q}\right)+\frac{1}{\sigma \cdot L_{s}} v_{c, s q} \\
& \frac{d i_{r d}}{d t}=\frac{1}{\sigma L_{s}}\left(\frac{M \cdot R s}{L_{s}} i_{s d}-\frac{1}{M} \omega i_{s q}-R_{r} i_{r d}+\left(\omega_{s} \sigma+\omega\right) L_{r} i_{r q}\right)+\frac{1}{\sigma \cdot L_{r}} v_{c, r d} \\
& \frac{d i_{r q}}{d t}=\frac{1}{\sigma L_{r}}\left(M \omega_{s q} \frac{M R s}{L_{s}} i_{s q}-\left(\omega_{s} \sigma+\omega\right) L_{r} i_{r d}-R_{r} i_{r q}\right)+\frac{1}{\sigma \cdot L_{r}} v_{c, r q} \\
& \frac{d \omega}{d t}=\frac{P^{2}}{J} \cdot M\left(i_{r d} i_{s d}-i_{r q} i_{s d}\right)-\frac{P}{J} C_{r}-\frac{f}{J} \omega
\end{aligned}
$$

With voltages $\left(v_{c, s d}, v_{c, s q}, v_{c, r d}, v_{c, r q}\right)$ as control variables. The tension of commende of park $\left(v_{s d}, v_{s q}, v_{r d}, v_{r q}\right)$. Can be deduced from the control voltages $\left(v_{c, s d}, v_{c, s q}, v_{c, r d}, v_{c, r q}\right)$ By the following relationships

$$
\begin{gathered}
v_{s d}=\frac{1}{\sigma}\left(v_{c, s d}+\frac{M}{L_{r}} v_{c, r d}\right) \\
v_{r d}=\frac{1}{\sigma}\left(\frac{M}{L_{s}} v_{c, s d}+v_{c, r d}\right) \\
v_{c, s d}=R_{s}\left(\sigma T_{s} . s+1\right) i_{s d}-\left(\frac{M \cdot R_{r}}{L_{r}} i_{r d}+\left(\omega_{s} \sigma-\omega(\sigma-1)\right) L_{s} i_{s q}+\omega M . i_{r q}\right) \\
\left.\begin{array}{c}
\text { From equations of the model }(12) \\
\text { control equations: }
\end{array}\right) \\
v_{c, s d}=R_{s}\left(\sigma T_{s} . s+1\right) i_{s q}-\left(\frac{M \cdot R_{r}}{L_{r}} i_{r q}+\left(\omega_{s} \sigma-\omega(\sigma-1)\right) L_{s} i_{s d}+\omega M . i_{r d}\right) \\
v_{c, s d}=R_{r}\left(\sigma T_{r} . s+1\right) i_{r d}-\left(\frac{M \cdot R_{s}}{L_{s}} i_{s d}+\frac{\sigma-1}{M} \omega \cdot L_{s} \cdot L_{r} \cdot i_{s q}+\frac{\omega_{s} \sigma-\omega}{L_{s}} i_{r q}\right) \\
v_{c, s d}=R_{r}\left(\sigma T_{r} . s+1\right) i_{r q}-\left(\frac{M \cdot R_{s}}{L_{s}} i_{s q}-\left(\omega_{s} \sigma+\omega\right) L_{r} i_{r d}-\omega M . i_{s d}\right)
\end{gathered}
$$

From equations of the model (12), we obtain the following

\subsection{Decoupling by Compensation}

The static compensation method consists in introducing terms, called equal compensation Fem, but with opposite signs so as to eliminate their influence and thus to separate the mutual actions on the two axes $\mathrm{d}$ and $\mathrm{q}$.
The model (15) can be re-expressed in the following form 


$$
\left\{\begin{array}{l}
v_{c, s d}=R_{s}\left(1+T_{s} \sigma \frac{d}{d t}\right) i_{s d}+F_{e m, s d} \\
v_{c, s d}=R_{s}\left(1+T_{s} \sigma \frac{d}{d t}\right) i_{s q}+F_{e m, s q} \\
v_{c, s d}=R_{r}\left(1+T_{r} \sigma \frac{d}{d t}\right) i_{r d}+F_{e m, r d} \\
v_{c, s d}=R_{r}\left(1+T_{r} \sigma \frac{d}{d t}\right) i_{r q}+F_{e m, r q}
\end{array}\right.
$$

The terms Fem, sd, Fem, sq, Fem, rd and Fem, rq correspond to the terms of coupling between the axes (d) and (q). with:

$$
\begin{gathered}
\left\{\begin{array}{l}
F_{e m, s d}=\frac{-M R_{r}}{L_{r}} i_{r d}-\omega_{s} \varphi_{s q}+\frac{M}{L_{r}} \omega_{r} \varphi_{r q} \\
F_{e m, s q}=\frac{-M R_{r}}{L_{r}} i_{r q}+\omega_{s} \varphi_{s d}-\frac{M}{L_{r}} \omega_{r} \varphi_{r d}
\end{array}\right. \\
\left\{\begin{array}{l}
F_{e m, r d}=\frac{-M R_{s}}{L_{s}} i_{s d}-\omega_{r} \varphi_{r q}+\frac{M}{L_{s}} \omega_{s} \varphi_{s q} \\
F_{e m, r q}=\frac{-M R_{r}}{L_{r}} i_{s q}+\omega_{r} \varphi_{r d}-\frac{M}{L_{s}} \omega_{s} \varphi_{s d}
\end{array}\right.
\end{gathered}
$$

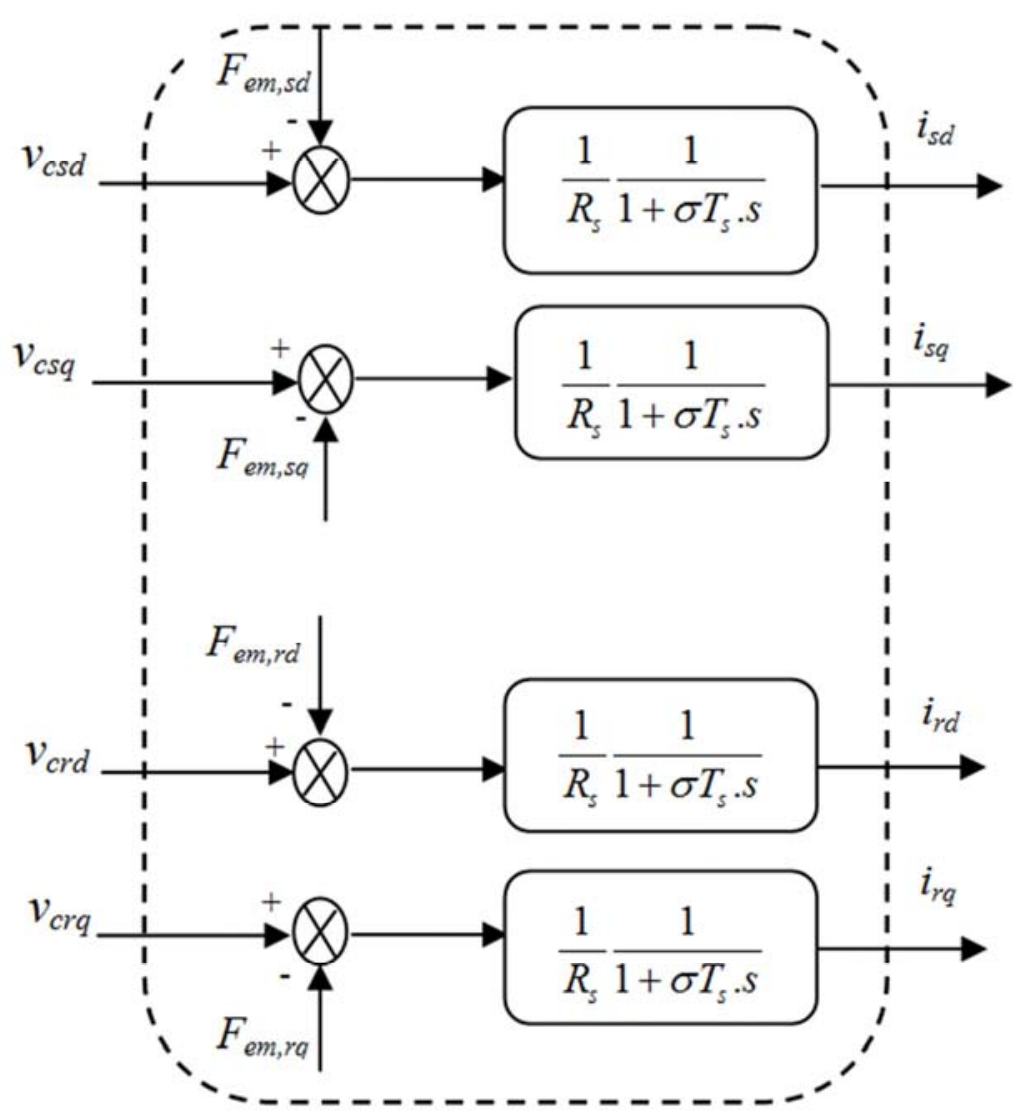

Figure 2. Model of the DFIM.

From equation (20) we obtain two transfer functions connecting the stator and rotor components of each axis:

With:
We can then represent the model (19) of the MADA by the following figure:

$$
\left\{\begin{array}{l}
v_{c, s d}=v_{1 s d}+F_{e m, s d} \\
v_{c, s q}=v_{1 s q}+F_{e m, s q} \\
v_{c, r d}=v_{1 r d}+F_{e m, r d} \\
v_{c, r q}=v_{1 r q}+F_{e m, r q}
\end{array}\right.
$$

$$
\left\{\begin{array}{l}
v_{1 s d}=\sigma L_{s} \frac{d i_{s d}}{d t}+R_{s} i_{s d} \\
v_{1 s q}=\sigma L_{s} \frac{d i_{s q}}{d t}+R_{s} i_{s q} \\
v_{1 s d}=\sigma L_{r} \frac{d i_{r d}}{d t}+R_{r} i_{r d} \\
v_{1 s d}=\sigma L_{r} \frac{d i_{r q}}{d t}+R_{r} i_{r q}
\end{array}\right.
$$


and

$$
\frac{i_{r d}(S)}{v_{1 r d}(s)}=\frac{i_{r q}(s)}{v_{1 r q}(s)}=\frac{1 / R_{r}}{1+\sigma \cdot T_{r} . S}
$$

These transfer functions are first-order and almost identical; each is a function of the machine parameters (rotor and stator respectively)

To obtain the different references of the currents to be regulated, we use the equations linking the following flows and currents:

$$
\left\{\begin{array}{l}
\phi_{s d}=L_{s} i_{s d}+M i_{r d} \\
\phi_{s q}=L_{s} i_{s q}+M i_{r q} \\
\phi_{r d}=L_{r} i_{r d}+M i_{s d} \\
\phi_{r q}=L_{r} i_{r q}+M i_{s q}
\end{array}\right.
$$

By imposing an orientation of the rotor flux and an operation at a unit power factor at the rotor we will have

$$
\left\{\begin{array}{l}
\phi_{r d}=\phi_{r} \\
\phi_{r q}=0
\end{array} \text { and } i_{r d}=0\right.
$$

We obtain the following system:

$$
\left\{\begin{array}{l}
\phi_{s d}=L_{s} i_{s d} \\
\phi_{s q}=L_{s} i_{s q}+M i_{r q} \\
\phi_{r d}=M i_{s d} \\
L_{r} i_{r q}=-M i_{s q}
\end{array}\right.
$$

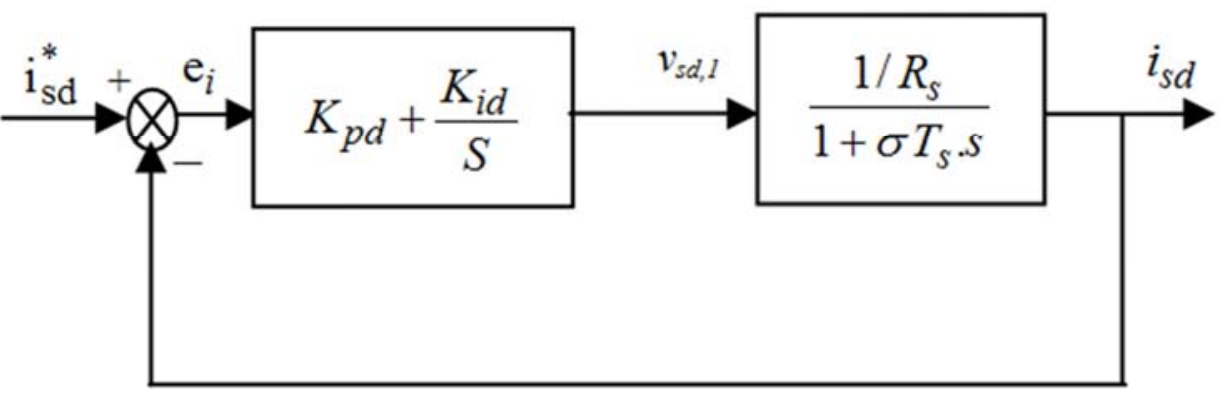

Figure 3. Block diagram for regulating stator currents.

In closed loop, we obtain a transfer function of the form

$$
G_{d F}(s)=\frac{i_{s d}}{i_{s d}^{*}}=\frac{\frac{1}{\sigma T_{s} R_{s}}\left(k_{p d} s+k_{i d}\right)}{s^{2}+\left(\frac{1+k_{p d} / R_{s}}{\sigma T_{s}}\right) s+\frac{k_{i d}}{\sigma T_{s} R_{s}}}
$$

Its characteristic polynomial highlights a second-order dynamics

$$
P(s)=s^{2}+\left(\frac{1+k_{p d} / R_{s}}{\sigma T_{s}}\right) s+\frac{k_{i d}}{\sigma T_{s} R_{s}}
$$

On the other hand, the expression of the electromagnetic torque becomes:

$$
C_{e m}=p \frac{M}{L_{r}} \phi_{r d} \cdot i_{s q}
$$

The different references of the currents to be regulated for an orientation of the rotor flux and a unit power factor operation at the rotor are

$$
\left\{\begin{array}{l}
i_{s d}^{*}=i_{s d-r e f}=\frac{1}{M} \phi_{r d-r e f} \\
i_{s q}^{*}=i_{s q-r e f}=\frac{L_{r}}{p M \phi_{r d-r e f}} C_{e m-r e f} \\
i_{r d}^{*}=i_{r d-r e f}=0 \\
i_{r q}^{*}=i_{r q-r e f}=-\frac{1}{p \phi_{r d-r e f}} C_{e m-r e f}
\end{array}\right.
$$

\subsection{Stator Current Regulation}

For currents, direct and quadrature, highlight two symmetric control loops equipped with regulators defined by the parameters $\left(K_{p d}, K_{i d}\right)$ and $\left(K_{p q}, K_{i q}\right)$ :

$$
\frac{i_{s d}}{v_{s d 1}}=\frac{i_{s q}}{v_{s q 1}}=\frac{1 / R_{s}}{1+\sigma T_{s} s}
$$

The block diagram of the direct current regulation is shown in the following figure

Let's impose two complex and conjugated poles with negative real parts for which the denominator of the corresponding transfer functions is of the form

$$
D(s)=s^{2}+2 \xi \omega_{0} s+\omega_{0}^{2}
$$

Thus, obtain the parameters of the corrector, according to the damping $\xi$ and the proper pulsation $\omega_{0}$

By identification between the equations (29) and (28), we obtain the following parameters of the PI corrector:

$$
\left\{\begin{array}{l}
K_{p d}=\left(2 \xi \omega_{0} \sigma T_{s}-1\right) R_{s} \\
K_{i d}=\omega_{0}^{2} \sigma T_{s} R_{s}
\end{array}\right.
$$


By choosing identical dynamics for the two current loops

$$
K_{p d}=K_{p q} \quad \text { and } \quad K_{i d}=K_{i q}
$$

\subsection{Rotor Current Regulation}

Recall the transfer function connecting the rotor components of each axis of the DFIM

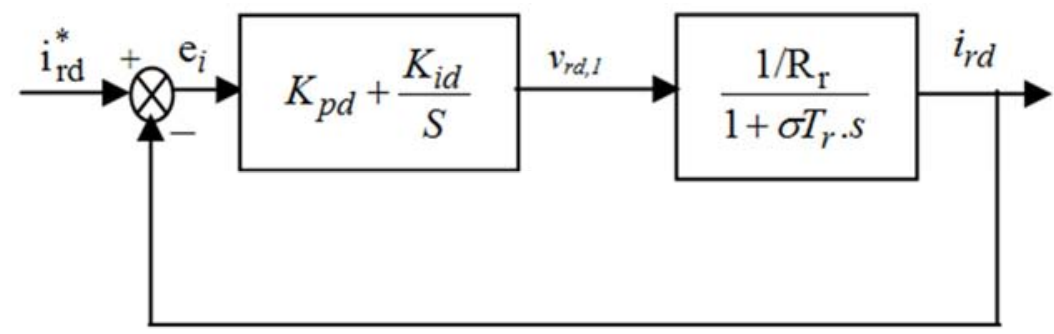

Figure 4. Block diagram for regulating rotor currents.

The closed loop transfer function is given as follows

$$
H_{I_{R}}(s)=\frac{I_{r d, q}}{I_{r d, q}{ }^{*}}=\frac{\frac{1}{\sigma T_{r} R_{r}}\left(K_{p I_{r}} \cdot S+K_{i, i_{r}}\right)}{s^{2}+S\left(\frac{1+K_{p i_{r}} / R_{r}}{\sigma T_{r}}\right)+\frac{K_{i, i_{r}}}{\sigma T_{r} R_{r}}}
$$

The same procedure performed for the correctors of the stator currents is applied to the correctors of the rotor currents. The parameters of the correctors are therefore the same. They are given by.

$$
K_{p I_{r}}=\left(2 \xi \omega_{0} \sigma T_{R}-1\right) R_{r} \text { et } K_{i, i_{r}}=\omega_{0}^{2} \sigma T_{r} R_{r}
$$

\subsection{Rotor Speed Regulation}

The external speed control loop will be defined by the

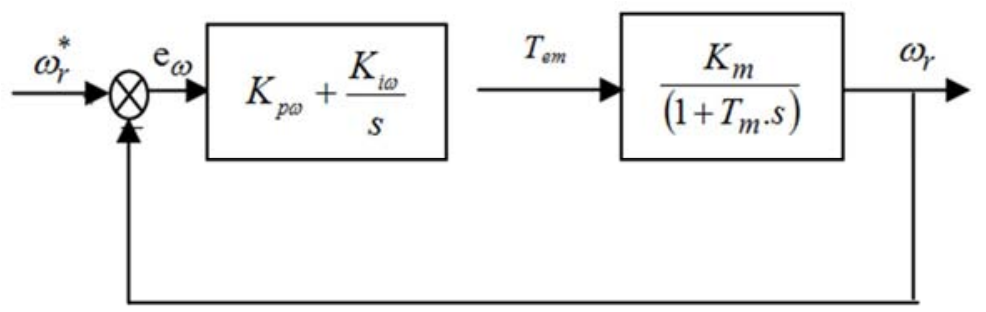

Figure 5. External speed control loop equipped with a PI regulator.

The closed loop transfer function is given by

$$
\frac{\omega}{\omega^{*}}=\frac{\frac{K_{m} \cdot K_{p \Omega} \cdot s+K_{m} \cdot K_{i \Omega}}{T_{m}}}{s^{2}+s \cdot\left(\frac{1+K_{m} \cdot K_{p \Omega}}{T_{m}}\right)+\frac{K_{m} \cdot K_{i \Omega}}{T_{m}}}
$$

By imposing the closed loop poles, obtain the parameters of the PI corrector: parameters $\left(K_{p \omega}, K_{i \omega}\right)$. From the equation of the mechanics governing the dynamics of rotating bodies

The relationship linking the speed to the electromagnetic torque is given by

$$
\begin{gathered}
\frac{\omega}{C_{e m}}=\frac{p}{\left(f_{v}+J . s\right)} \\
\frac{\omega}{C_{e m}}=\frac{K_{m}}{\left(1+T_{m} . s\right)}
\end{gathered}
$$

Where $k_{m}=\frac{p}{f}, \quad T_{m}=\frac{J}{f}$

The block diagram of the speed loop regulation is in Figure 5:

$$
\begin{gathered}
K_{p \omega}=\left(2 \xi \omega_{0} T_{m}-1\right) / K_{m} \\
K_{i \omega}=\omega_{0}{ }^{2} T_{m} / K_{m}
\end{gathered}
$$

\section{Estimation of the Speed by the Technique of MRAS}

The adaptive reference model system is based on the comparison of the outputs of the two estimators. The first, 
which does not introduce the magnitude to be estimated (the speed in our case), is called reference model and the second is the adjustable model. The error between these two models drives an adaptation mechanism that generates the speed by applying Popov's criterion of hyper-stability. This speed is used in the adjustable model, [11].

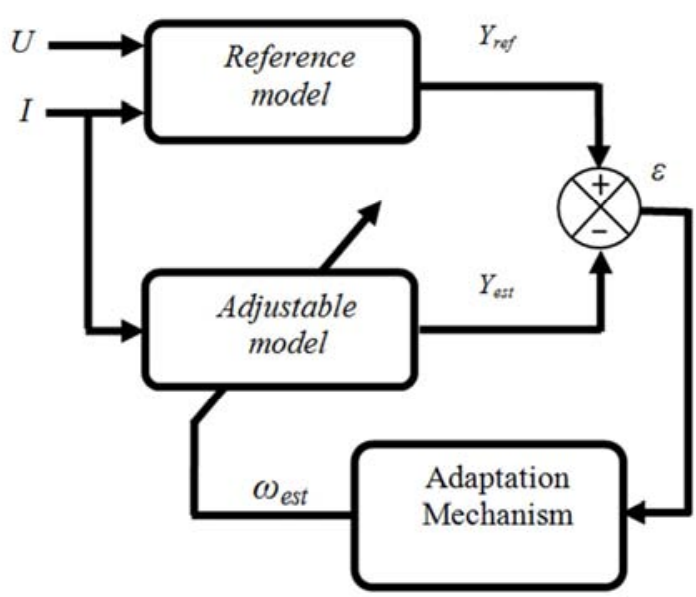

Figure 6. Basic configuration of the MRAS method.

$$
\frac{d}{d t} \varphi_{r e f}-\frac{d}{d t} \hat{\varphi}_{r}=\frac{d}{d t} e
$$

Application of a MRAS based on rotor flux to DFIM Reference Model:

Based on the voltage equations and equations of the stator and rotor flux of the machine model in the fixed reference $(\alpha, \beta)$, can write

$$
\begin{gathered}
v_{s \alpha \beta}=R_{s} i_{s \alpha \beta}+\left(\frac{d \varphi_{s \alpha \beta}}{d t}\right) \\
\phi_{s \alpha \beta}=L_{s} i_{s \alpha \beta}+M i_{r \alpha \beta} \\
\phi_{r \alpha \beta}=L_{r} i_{r \alpha \beta}+M i_{s \alpha \beta}
\end{gathered}
$$

From Equation (41), we have

$$
i_{r \alpha \beta}=\frac{1}{M}\left(\phi_{r \alpha \beta}-L_{s} i_{s \alpha \beta}\right)
$$

replace this equation in (42) while deriving the result, we obtain:

$$
\frac{d \phi_{r \alpha \beta}}{d t}=\frac{L_{r}}{M} \frac{d \phi_{s \alpha \beta}}{d t}+\left(M-\frac{L_{r} L_{s}}{M}\right) \frac{d i_{s \alpha \beta}}{d t}
$$

And from (40), we find:

$$
\frac{d \varphi_{s \alpha \beta}}{d t}=v_{s \alpha \beta}-R_{s} i_{s \alpha \beta}
$$

Replace (45) in (44), we get

$$
\frac{d \phi_{r \alpha \beta}}{d t}=\frac{L_{r}}{M}\left(v_{s \alpha \beta}-R_{s} i_{s \alpha \beta}\right)+\left(M-\frac{L_{r} L_{s}}{M}\right) \frac{d i_{s \alpha \beta}}{d t}
$$

Finally, can write the equations of this reference model as follows

$$
\left\{\begin{array}{l}
\frac{d \phi_{r \alpha}}{d t}=\frac{L_{r}}{M}\left(v_{s \alpha}-R_{s} i_{s \alpha}-\sigma L_{s} \frac{d i_{s \alpha}}{d t}\right) \\
\frac{d \phi_{r \beta}}{d t}=\frac{L_{r}}{M}\left(v_{s \beta}-R_{s} i_{s \beta}-\sigma L_{s} \frac{d i_{s \beta}}{d t}\right)
\end{array}\right.
$$

The Adaptive model

Using the equations of the rotor voltages, one can write:

$$
\left\{\begin{array}{l}
\frac{d}{d t} \phi_{r \alpha}=v_{r \alpha}-\frac{1}{T_{r}} \phi_{r \alpha}-\omega \cdot \phi_{r \beta}+\frac{M}{T_{r}} i_{s \alpha} \\
\frac{d}{d t} \phi_{r \beta}=v_{r \beta}-\frac{1}{T_{r}} \phi_{r \beta}+\omega . \phi_{r \alpha}+\frac{M}{T_{r}} i_{s \beta}
\end{array}\right.
$$

It is clear that the speed appears explicitly in (48), For estimated flux and speed, such that the current of the stator will be considered as measurable input the system (48) is written:

$$
\left\{\begin{array}{l}
\frac{d}{d t} \hat{\phi}_{r \alpha}=v_{r \alpha}-\frac{1}{T_{r}} \hat{\phi}_{r \alpha}-\hat{\omega} \cdot \hat{\phi}_{r \beta}+\frac{M}{T_{r}} i_{s \alpha} \\
\frac{d}{d t} \hat{\phi}_{r \beta}=v_{r \beta}-\frac{1}{T_{r}} \hat{\phi}_{r \beta}+\hat{\omega} \cdot \hat{\phi}_{r \alpha}+\frac{M}{T_{r}} i_{s \beta}
\end{array}\right.
$$

The adaptation algorithm is chosen so as to converge the adjustable model to the reference model by minimizing the error and having a stability of the system.

\section{Adaptation mechanism}

The input of this mechanism is activated by the error between $\varphi_{\text {ref }}$ and $\hat{\varphi}_{r}$. Make the difference between the reference model and the adjustable model, it comes

After the calculation get

$$
\frac{d}{d t} e=\left(-\frac{1}{T_{r}}+j \hat{\omega}\right) e+j(\omega-\hat{\omega}) \hat{\varphi}_{r}
$$

The previous equation can be expressed as follows

$$
\left[\begin{array}{l}
\frac{d}{d t} e_{\alpha} \\
\frac{d}{d t} e_{\beta}
\end{array}\right]=\left(\begin{array}{cc}
-\frac{1}{T_{r}} & -\omega \\
\omega & -\frac{1}{T_{r}}
\end{array}\right)\left[\begin{array}{l}
e_{\alpha} \\
e_{\beta}
\end{array}\right]+(\omega-\hat{\omega})\left[\begin{array}{l}
-\hat{\varphi}_{r \alpha} \\
+\hat{\varphi}_{r \beta}
\end{array}\right]
$$

Or

$$
\dot{e}=[A] \cdot e-[W]
$$

with 


$$
[A]=\left(\begin{array}{cc}
-\frac{1}{T_{r}} & -\omega \\
\omega & -\frac{1}{T_{r}}
\end{array}\right),[W]=(\omega-\underline{\hat{\omega}}) \hat{\underline{\varphi}}_{r}
$$

To ensure the convergence of the system, Schauder proposed an adaptation law that satisfies Popov's stability criterion given by the relation

$$
\begin{gathered}
\hat{\omega}=k_{p} .\left(\varphi_{r \beta} \hat{\varphi}_{r \alpha}-\varphi_{r \alpha} \hat{\varphi}_{r \beta}\right)+k{ }_{i}\left(\varphi_{r \beta} \hat{\varphi}_{r \alpha}-\varphi_{r \alpha} \hat{\varphi}_{r \beta}\right) d t \\
e=\varphi_{r \beta} \hat{\varphi}_{r \alpha}-\varphi_{r \alpha} \hat{\varphi}_{r \beta}
\end{gathered}
$$

The structure of the mechanical sensorless control of the DFIM by rotor flux orientation according to the rotor fluxbased MRAS principle is shown in Figure 8 and the one used for the simulation.

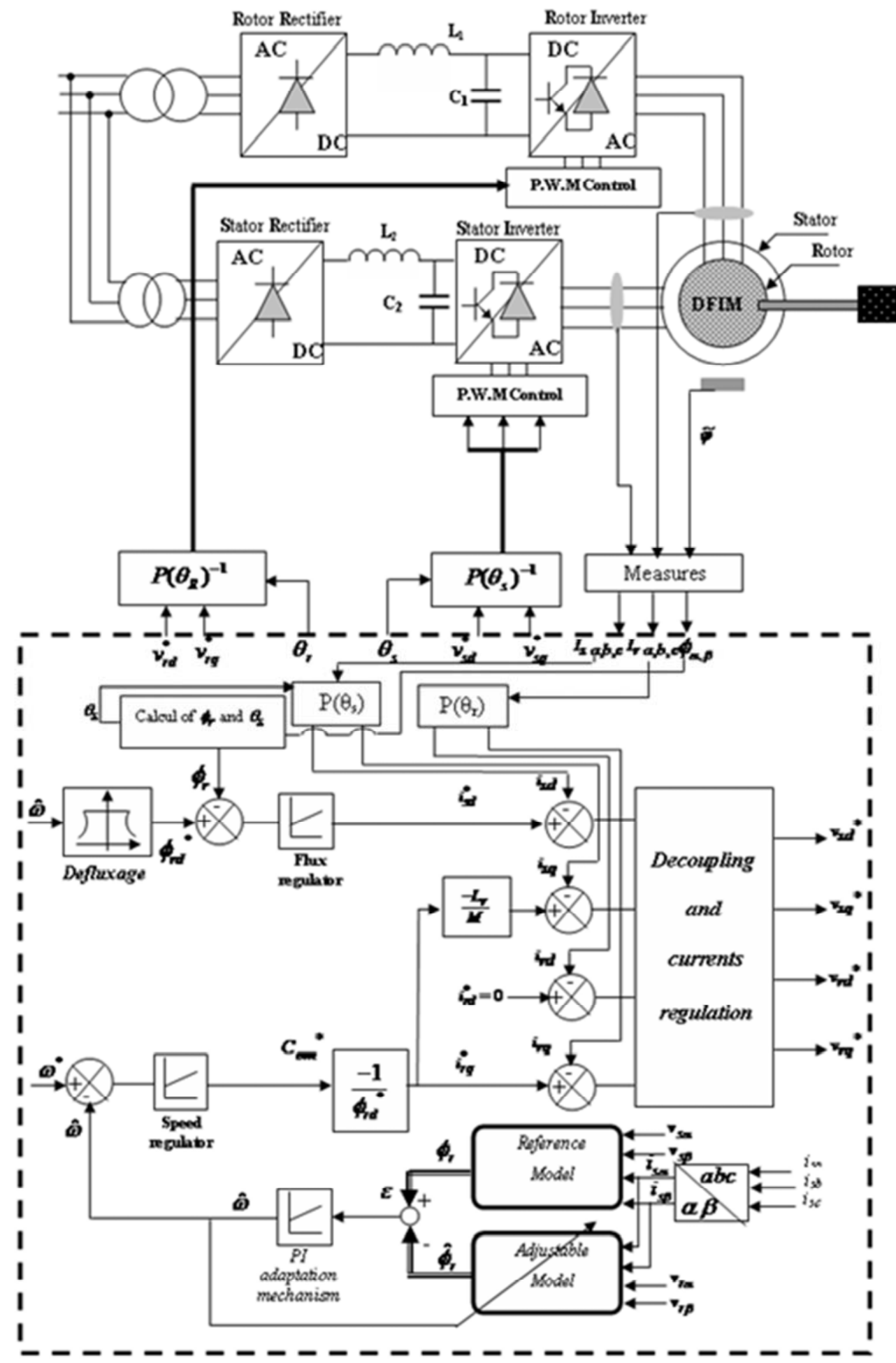

Figure 7. Mechanical sensorless control of MADA based on MRAS. 

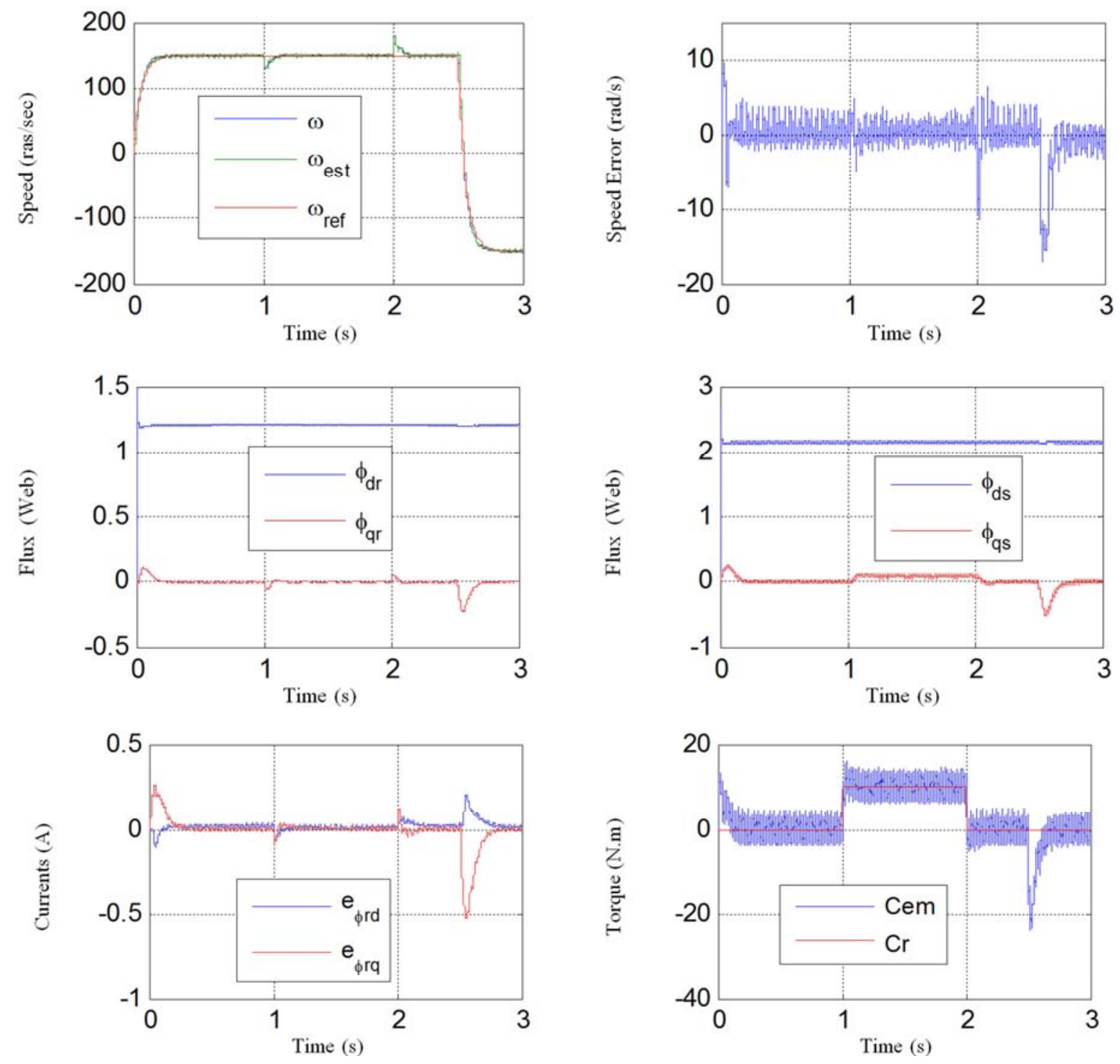

Figure 8. Sensorless speed control using MRAS estimator.

\section{Simulation Results and Discussion}

Simulated the system for a speed reference of $150 \mathrm{rad} / \mathrm{s}$, under the application of a load torque equal to $10 \mathrm{Nm}$ between instants $\mathrm{t} 1=1 \mathrm{~s}$ and $\mathrm{t} 2=2 \mathrm{~s}$ respectively and reversal of direction of rotation to instant $\mathrm{t}=2.5 \mathrm{~s}$ to $-150 \mathrm{rad} / \mathrm{s}$. Got the results above (Figure 8)

With regard to the simulation results, the actual speed perfectly follows the estimated speed with a low tracking error during the transient phases and canceling out in steady state but during the transition from $150 \mathrm{ras} / \mathrm{s}$ to $-150 \mathrm{rad} / \mathrm{s}$ that there is an estimation error. An excellent orientation of the rotor flux on the direct axis is also observed. This affects the electromagnetic torque. During the changes of the setpoints, and in particular during the inversion of rotation, the change of direction of the torque does not degrade the orientation of the flow. Likewise for the estimated components of the rotor flux that are little influenced by this inversion of speed.

Good sensitivity to load disturbances is observed, with a relatively low rejection time.

Influence of parametric variations

In order to study the influence of parametric variations on the behavior of vector control without speed sensor based on the MRAS technique, we introduced a variation of $+50 \%$ of $\mathrm{Rr}$ in the first test, then a variation of $+50 \%$ of Rs. That obtained the results as shown in Figure 9 and 10, respectively:

It should be noted that during the variation of the rotor resistance $\mathrm{Rr}$ all the magnitudes of the machine present a small disturbance (Figure 9).

For a nominal value of $\mathrm{Rr}$, the stator resistance is increased by $+50 \%$ of its nominal value, the results of the figure 10 are obtained. These results prove that the variation of Rs further deteriorates the accuracy of the estimate, especially in the low-speed zone "-150 rad / s", will lead to a significant estimation error plus the instability of the estimator. the high speed zone the estimation error remains low. It conclude that this technique is very sensitive to variations in machine parameters. 

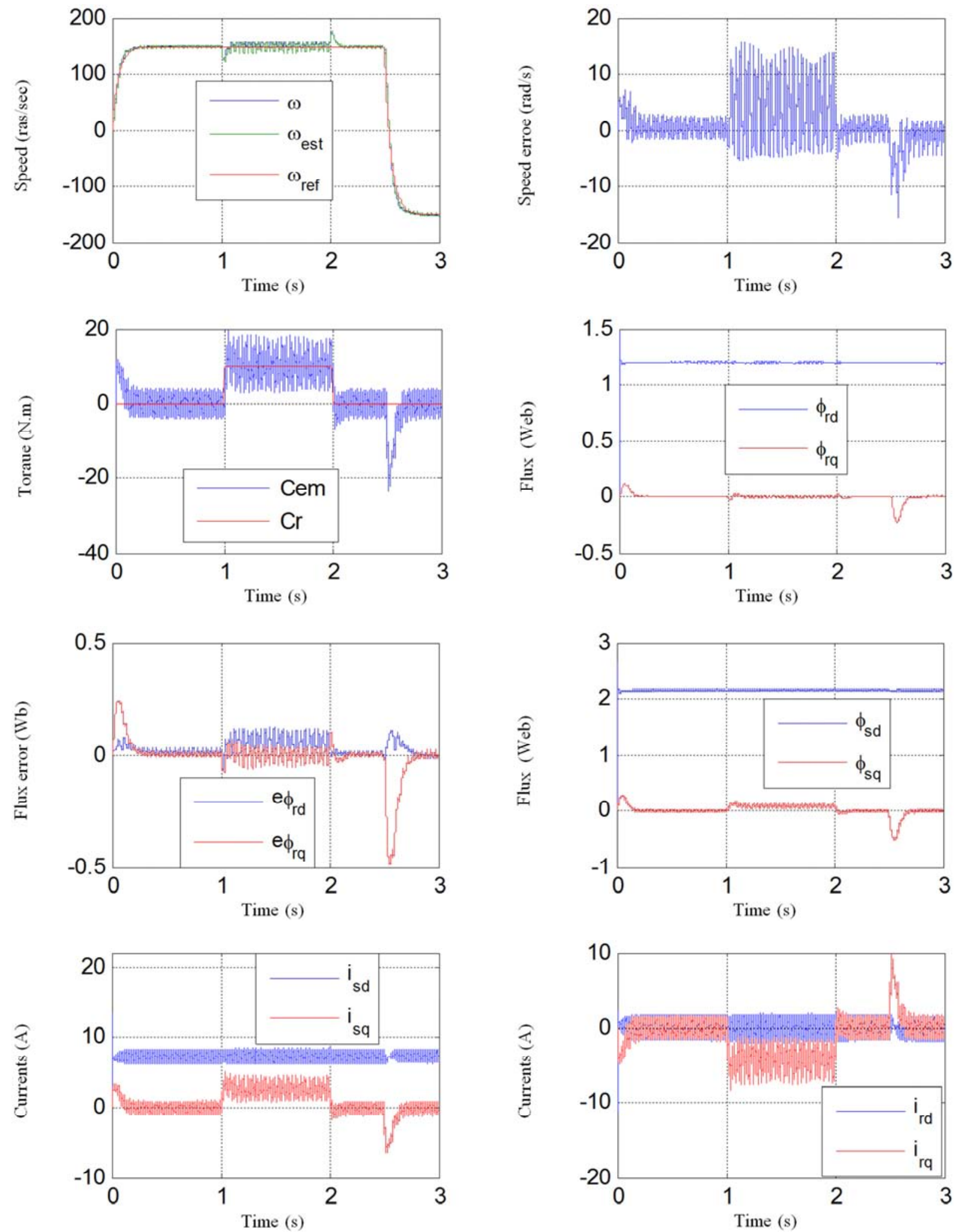

Figure 9. Sensorless speed control using MRAS estimator during variation of $+50 \%$ of Rr. 

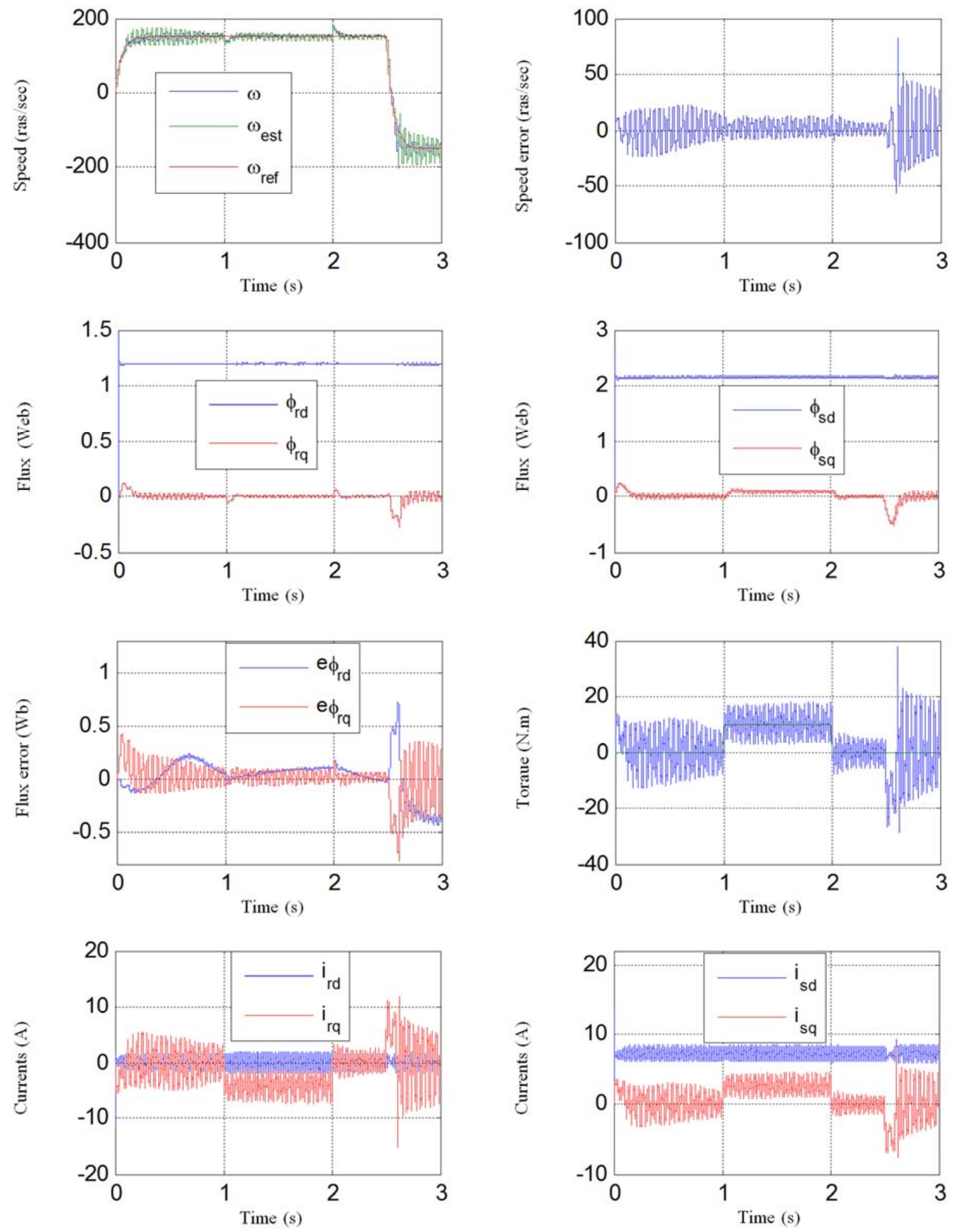

Figure 10. Sensorless speed control using MRAS estimator during variation of $+50 \%$ of Rs.

\section{Conclusion}

In this article, we have exploited MRAS technique for DFIM speed estimation.

A numerical simulation in the Matlab-Simulink environment was performed to validate these performances.
From the simulation results obtained, it can be concluded that this proposed estimation technique is valid for the nominal conditions. On the other hand, the studied estimator has a good robustness with respect to the variation of the load and the pursuit, makes it possible to reach good functional performances with a low cost and low volume installation.

By contrast, the MRAS technique is not robust to 
parametric variations to different degrees, such as rotor and stator resistance. For this purpose, the online adaptation of these parameters is essential to solve this problem.

\section{Appendix}

Induction Motor Parameters

$50 \mathrm{~Hz}, 1.5 \mathrm{Kw}, 1450 \mathrm{rpm}, \mathrm{Rr}=1.68 \Omega, \mathrm{Rs}=1.75 \Omega$,

Ls $295=\mathrm{mH}$, Lr 104= mH, M=165 $\mathrm{mH}$

$\mathrm{J}=0.01 \mathrm{~kg} . \mathrm{m} 2, \mathrm{~F}=0.0027 \mathrm{~kg} \cdot \mathrm{m} 2 / \mathrm{s}$

\section{References}

[1] S. Drid, M. Tadjine and M. S. Naît-Saîd, "Robust backstepping vector control for the doubly fed induction motor," The Institution of Engineering and Technology, Control Theory Appl., vol. 1, no. 4, pp. 861-868, 2007.

[2] D. Ben Attous and Y. Bekakram "Speed Control of a Doubly Fed Induction Motor using Fuzzy Logic Techniques", International Journal on Electrical Engineering and Informatics - Volume 2, Number 3, 2010.

[3] S. Drid, M. Tadjine, M. S. Nait-Said, "Nonlinear feedback control and torque optimization of a doubly fed induction motor," Journal of Electrical Engineering, Vol. 56, NO. 3-4, 2005, 57-63.

[4] K. Loukal, L. Benalia, A. Bouguerra, "Speed Control of a Doubly Fed Induction Motor Based on Fuzzy GainAdaptive IP", International Journal of Information Technology and Electrical Engineering 2012.
[5] DJ. CHERIFI, Y. MILOUD, "A Luenberger State Observer for Stator Resistance Estimation in Sensorless Induction Motor Drive", International Review on modelling and simulation (IREMOS), April 2013.

[6] Khojet El Khil, I. Slama-Belkhodja, M. Pietrzak-David and B. De Fornel. "Power distribution law in a Doubly Fed Induction Machine". Mathematics and Computers in Simulation, volume 71, pages 360-368, 2006.

[7] P. E. Vidal, D. Pietrzak and M. B. de Fornel, "StatorFlux Oriented Control Of a Doubly Fed InductionMachine", 10th European Conference on Power Electronics and Applications, Toulouse, France, pp. 1-9, 2003.

[8] Y. Bekakra and D. Ben Attous, " Comparison between Fuzzy Sliding Mode and Traditional IP Controllers in a Speed Control of a Doubly Fed Induction Motor", ECTI TRANSACTIONS ON ELECTRICAL ENG., ELECTRONICS, AND COMMUNICATIONS VOL. 10, NO. 2 August 2012.

[9] DJ. CHERIFI, Y. MILOUD, "New Fuzzy Luenberger Observer for Performance Evaluation of a Sensorless Induction Motor Drive", International Review of Automatic Control, Theory and Applications (IREACO), July 2013 (Vol. 6 N. 4).

[10] G. SALLOUM, R. MBAYED, M. DE FORNEL B. PIETRZAK-DAVID, "Mixed Sensitivity $\mathrm{H} \infty$ Control of Doubly Fed Induction Motor," pp 1300-1304, 1-4244-07559/07/\$20.00 C2007 IEEE, pp 1300-1304.

[11] DJ. CHERIFI, Y. MILOUD, "performance evaluation of a sensorless induction motor drive using a mras speed observer", JOURNAL OF CURRENT RESEARCH IN SCIENCE, 2013. 